\title{
Immunomodulatory Effect of Imiquimod Through CCL22 Produced by Tumor-associated Macrophages in B16F10 Melanomas
}

\author{
SADANORI FURUDATE*, TAKU FUJIMURA*, YUMI KAMBAYASHI, \\ AYA KAKIZAKI, TAKANORI HIDAKA and SETSUYA AIBA
}

\author{
Department of Dermatology, Tohoku University Graduate School of Medicine, Sendai, Japan
}

\begin{abstract}
Background/Aim: Tumor-associated macrophages (TAMs), together with splenic CD11 ${ }^{+}$cells, help maintain the tumor microenvironment. The immunomodulatory compound imiquimod (IQM) stimulates innate immune cells, including macrophages, to induce antitumor effects. In order to elucidate the effects of IQM on the tumor microenvironment, we investigated the immunomodulatory effect of IQM during melanoma growth by using the B16F10 melanoma model. Materials and Methods: To elucidate the immunomodulatory effects of IQM on the tumor microenvironment, we isolated $C D 11 b^{+}$TAMs and splenic CD11 ${ }^{+}$cells and evaluated the immunomodulatory effects of IQM, using the B16F10 melanoma model. Results: IQM suppressed B16F10 melanoma growth in parallel with reduction of Foxp $3^{+}$regulatory $T$ cells (Tregs) at the tumor site, caused by the down-regulation of CCL22 production by tumor-derived and splenic CD11b ${ }^{+}$cells. Subsequently, we investigated the antitumor or tumor-loading effects of splenic CD11b ${ }^{+}$cells on B16F10 melanoma growth in vivo. B16F10 melanoma growth was accelerated by splenic $C D 11 b^{+}$cells from untreated mice, but was inhibited by splenic $\mathrm{CD}_{11 b^{+}}$cells from IQM-treated mice. Consistent with these results, Foxp $3^{+}$Tregs were significantly decreased in tumors of mice implanted with both melanoma and splenic $C D 11 b^{+}$ cells from topical IQM-treated mice. Furthermore, intratumoral administration of anti-CCL22 antibody inhibited B16F10 melanoma growth by decreasing Treg recruitment at
\end{abstract}

This article is freely accessible online.

*These Authors contributed equally to this study.

Correspondence to: Taku Fujimura, MD, Ph.D., Department of Dermatology, Tohoku University Graduate School of Medicine, 11 Seiryo-machi, Aoba-ku, Sendai, Miyagi 980-8574, Japan. Tel: +81 227177271, Fax: +81 227177361, e-mail: tfujimura1@mac.com

Key Words: Tumor-associated macrophages, splenic CD11 b cells, regulatory $\mathrm{T}$ cells, melanoma, imiquimod. the tumor site. Conclusion: Our results suggest a possible mechanism for the antitumor immune response induced by IQM through tumor-associated macrophages.

Imiquimod (IQM) is an immunomodulatory, small-molecule compound in the imidazoquinoline family that induces antitumor effects through Toll-like receptor 7 (TLR7). As a TLR7 agonist, IQM stimulates innate immunity, including tumor-associated macrophages (TAMs) (1-3). In an experimental murine model, the addition of IQM to cryosurgery increased the cellular immune response against tumor antigens, leading to complete rejection of B16OVA melanoma (4). Drobits et al. reported that the anti-melanoma effect of IQM is dependent on the recruitment of plasmacytoid dendritic cells (DCs) to the skin through chemokine (C-C motif) ligand 2 (CCL2) produced by mast cells (2). Notably, CCL2 also recruits immature myeloid cells to the tumor microenvironment $(5,6)$. Singh et al. reported the therapeutic effects of a TLR7/8 dual agonist (3M-052) on $\mathrm{B} 16 \mathrm{~F} 10$ melanoma in vivo. They concluded that intratumoral administration of 3M-052 significantly suppressed B16F10 melanoma growth by increasing CCL2 production from the tumor microenvironment, which might result in induction of M1 macrophages, and even enhanced the therapeutic effect of immune checkpoint inhibitors, such as anti-CTLA4 antibody (Ab) and anti-PD-L1 Ab (7).

In humans, IQM is clinically effective for the treatment of superficial melanomas such as lentigo maligna $(8,9)$. Several reports have suggested that IQM may be an optimal reagent for invasive melanoma when used in combination with other therapies $(3,4,7,10,11)$. Turza et al. reported that IQM was effective in 10 cases of superficial dermal and subcutaneous metastasis of melanoma when used in combination with intralesional interleukin (IL)-2 (9). They concluded that IQM could be used to control dermal metastatic melanoma, but that intralesional IL-2 is indispensable for controlling subcutaneous melanoma, suggesting that the abrogation of regulatory $\mathrm{T}$ cell (Treg) function is necessary for induction of an antitumor immune response by IQM (9). 
CCL22 attracts chemokine (C-C motif) ligand 4 (CCR4) ${ }^{+}$ $\mathrm{T}$ cells, including Tregs, in the lesional skin of melanoma (12). As Klarquist et al. reported, CCL22-related Tregs drive B16F10 melanoma growth in vivo, and the diversion of Tregs to normal skin by CCL22 vaccination could limit tumor-infiltrating Tregs, subsequently suppressing melanoma growth (12). In addition, as we previously reported, immunomodulatory drugs such as interferons (IFNs) could modulate CCL22 production from TAMs to decrease Treg recruitment to the tumor (13). Moreover, another CCR4 ligand, CCL2, is highly produced in the advanced stage of ret mouse melanoma (14), suggesting the significance of Treg-related chemokines in melanoma development. These findings suggested that reduction of tumor-derived chemokines could be a therapeutic target for melanoma. In the present study, we examined the therapeutic and immunomodulatory effects of topical IQM on established B16F10 melanomas in vivo, focusing on its effects on TAMs, splenic $\mathrm{CD}_{11 \mathrm{~b}^{+}}$cells and Tregs.

\section{Materials and Methods}

Ethics statement for animal experiments. The protocol for the animal study was approved by the ethics committee of Tohoku University Graduate School of Medicine for Animal Experimentation, Sendai, Japan (Permit number: 2014-153). The research practices comply with the Tohoku University Graduate School of Medicine's Animal Experimentation Ethics guidelines and policies. All surgeries were performed under sodium pentobarbital anesthesia, and all efforts were made to minimize suffering.

Animals and the melanoma cell line. C57BL/6 mice and BALB/c mice (5 to 8 weeks old) were purchased from Japan Shizuoka Laboratory Animal Center (Shizuoka, Japan) and housed in the animal facility at the Tohoku University Graduate School of Medicine. The murine melanoma cell line, B16F10, was obtained from the American Type Culture Collection (Manassas, VA, USA) and cultured in Dulbecco's minimal essential medium (Sigma, Tokyo, Japan) supplemented with $10 \%$ heat-inactivated fetal calf serum (FCS) (Invitrogen, Tokyo, Japan). All mice were bred under specific pathogen-free conditions at the Tohoku University Graduate School of Medicine.

Reagents. Imiquimod cream (5\%) was a kind gift from Mochida Pharmaceutical Co., Ltd (Tokyo, Japan). Blocking monoclonal Ab against mouse CCL22 (158132) was purchased from R\&D Systems (Minneapolis, MN, USA), and rat IgG was purchased from Syn Corporation Ltd (Kyoto, Japan). We used the following Abs for flow cytometric (FACS) analysis: rat monoclonal Abs for mouse CD4 (L3T4) APC (BD Bioscience, Tokyo, Japan), mouse CD8 (53-6.7) APC (BD Bioscience), mouse CD11b (M1/70) APC (BD Bioscience), mouse CD69 (H1.2F3) PE (BD Bioscience, Tokyo, Japan), mouse PD-1 (RMP1-30) PE (BD Bioscience), mouse PDL1 (MIH5) (BD Bioscience), mouse CD45 (A20) PE (BD Bioscience) and an anti-Foxp3 APC staining kit. Respective isotype controls were purchased from eBioscience (San Diego, CA, USA). Collagenase type 4 was purchased from Cell Systems (Troisdorf,
Germany). DNase1 and lipopolysaccharide (LPS) were purchased from Sigma (Tokyo, Japan).

Tumor inoculation and treatment. B16F10 melanoma cells (100 $\mu \mathrm{l}$ of $2 \times 10^{6}$ cells $/ \mathrm{ml}$ ) were subcutaneously injected into female C57BL/6 mice as described previously (13). For quantitative real-time PCR (qRT-PCR) and FACS analysis, 5\% imiquimod cream was topically administered on day 7, and the tumor was harvested on day 9. For qRT-PCR, whole tumors were frozen with liquid nitrogen and then crushed with a Cryo-Press (Microtec, Chiba, Japan). Total RNA was extracted by using ISOGEN (Nippon Gene, Tokyo, Japan) according to the manufacturer's instructions. For FACS analysis, whole tumors were incubated with collagenase 4 and DNase I for $20 \mathrm{~min}$ at $37^{\circ} \mathrm{C}$, followed by mechanical disruption, as previously described (13). After single-cell suspensions were obtained, FACS analysis was performed. A total of $1 \times 10^{5}$ cells were re-suspended in PBS/1\% FCS and incubated with a combination of Abs for $30 \mathrm{~min}$ at $4^{\circ} \mathrm{C}$. For the detection of Foxp3 expression, cells were fixed and permeabilized by using Cytofix/Cytoperm solution (BD Bioscience) according to the manufacturer's protocol. After washing, the cells were kept in PBS $/ 1 \%$ FCS. For all assays, cells were analyzed by using a C6 flow cytometer (Accuri Cytometers Inc., Ann Arbor, MI, USA).

For the therapeutic experiments, the size of established tumors was measured with a caliper (Mitutoyo, Utsunomiya, Japan), and tumor volume was estimated by using the formula: $\pi / 6 \times$ length $\times$ width2. Starting on day 6 , imiquimod cream $(5 \%)$ was topically administered to the tumors three times a week. Tumor-bearing animals were sacrificed when the tumors displayed severe ulceration or reached a size of $1,000 \mathrm{~mm}^{3}$.

$R N A$ extraction and quantitative real-time PCR experiments. Total RNA was extracted by using an RNeasy Micro kit (Qiagen, Courtaboeuf, France), according to the manufacturer's instructions. RNA was eluted with $14 \mu \mathrm{l}$ of RNase-free water. Contaminating genomic DNA was removed with DNase I treatment (RNase-Free DNase Set; Qiagen). Reverse transcription was performed with the SuperScript VILO cDNA synthesis kit (Invitrogen). Amplification reactions were performed by using an Mx 3000P real-time quantitative PCR system (Stratagene, Tokyo, Japan). Relative mRNA expression levels were calculated for each gene and each time point after normalization against GAPDH by using the $\Delta \Delta \mathrm{Ct}$ method. The experiments were repeated at least five times under identical conditions. The results are shown as the mean \pm standard deviation (SD) of all experiments.

Purification of $\mathrm{CD}_{11 b^{+}}$cells. $\mathrm{CD} 11 \mathrm{~b}^{+}$cells were isolated from tumors and spleens by using MACS beads (Miltenyi, BergischGladbach, Germany), as previously reported (15). Briefly, tumors were incubated with collagenase 4 and DNase I for 20 min at $37^{\circ} \mathrm{C}$, followed by mechanical disruption. After single-cell suspensions were obtained, dead cells were depleted by using a dead cell removal kit from Miltenyi, according to the manufacturer's protocol. Thereafter, aliquots of $10^{7}$ cells were incubated for $15 \mathrm{~min}$ with $10 \mu \mathrm{l}$ of anti-CD11b beads (clone M1/70.15.11.5) (Miltenyi) in $100 \mu \mathrm{l}$ MACS buffer (PBS, $0.5 \mathrm{mmol}$ EDTA, $0.5 \% \mathrm{BSA}$ ) at $4^{\circ} \mathrm{C}$, washed twice with MACS buffer and subjected to two consecutive rounds of separation via magnetic MS columns (Miltenyi). This procedure yielded predominantly $\mathrm{CD} 11 \mathrm{~b}^{+}$cells with purity greater than $80 \%$ from tumors and greater than $95 \%$ from spleens, as assessed by FACS analysis. 
$T$ cell proliferation assays. Bone marrow-derived dendritic cells (BMDCs) $\left(5 \times 10^{3}\right.$ cells/well) generated from BALB/c mice were dispensed in 96-well round-bottom plates (15). Thereafter, MACS bead isolated responder $\mathrm{CD} 4+\mathrm{T}$ cells from spleens and lymph nodes $\left(1 \times 10^{5}\right.$ cells/well $)$ and graded doses of $\mathrm{CD} 11 \mathrm{~b}^{+}$cells were added. Plates were incubated at $37^{\circ} \mathrm{C}$ for 4 days. The cultures were pulsed with $\left[{ }^{3} \mathrm{H}\right]$ thymidine $(20 \mu \mathrm{Ci} /$ well $) 16 \mathrm{~h}$ prior to harvesting. Incorporated radioactivity was determined using a beta-plate (Wallac, Turku, Finland).

Chemokine and cytokine enzyme-linked immunosorbent assays (ELISAs). CD11b $\mathrm{b}^{+}$cells from the tumors and spleens of each group were cultured with LPS $(0.5 \mu \mathrm{g} / \mathrm{ml})$ in 96 -well flat-bottom plates. After $24 \mathrm{~h}$ of culture, the supernatants were collected. Secretion of CCL17, CCL22, CXCL9, CXCL10, CXCL11, IFN- $\gamma$ or IL-10 was determined by ELISA (R\&D Systems), according to the manufacturer's instructions. The experiments were repeated at least three times under identical conditions, and the results shown are representative of all independent experiments.

Assessment of the suppressive function of splenic CD11b+ cells in vivo. $\mathrm{CD} 11 \mathrm{~b}^{+}$cells $\left(2 \times 10^{5}\right.$ cells $)$ isolated from the spleens of each treated group were mixed with B16F10 melanoma cells $\left(2 \times 10^{5}\right.$ cells) and subcutaneously injected into female C57BL/6 mice. For the CCL22 blockade, anti-mouse CCL22 Ab $(0.1 \mathrm{mg})$ or control $\operatorname{IgG} 2 \mathrm{a}(0.1 \mathrm{mg})$ was administered intratumorally $(0.1 \mathrm{mg})$ on days 6,10 and 13 after tumor inoculation. The size of established tumors was measured with a caliper (Mitutoyo), and the volume was calculated by using the formula: $\pi / 6 \times$ length $\times$ width $^{2}$. All assays comprised of 5 mice in each group and were performed at least three times. The results shown are a mean of all experiments.

Statistical analysis. For a single comparison of two groups, the unpaired Student's $t$-test was used. $p$-Values $<0.05$ were considered statistically significant.

\section{Results}

IQM decreased Treg recruitment, leading to the suppression of B16F10 melanoma growth. Localized TLR7/8 activation induces antitumor immunity and partially suppresses B16F10 melanoma growth in vivo $(4,7,11)$. To confirm the potential therapeutic effect of IQM, we first examined the effect of IQM on murine B16F10 melanoma growth in vivo. Topical IQM significantly suppressed B16F10 melanoma growth in vivo as compared to untreated melanoma (Figure 1A). To evaluate the possible mechanism for the therapeutic effects of topical IQM, we evaluated the number of Tregs in the tumors by flow cytometry, as we previously described (13). Whole-tumor cells were analyzed $48 \mathrm{~h}$ after stimulation with or without topical IQM. The percentage of Foxp3 ${ }^{+}$Tregs within the $\mathrm{CD}^{+} \mathrm{T}$ cell population was significantly decreased by topical administration of IQM compared to untreated mice $(p<0.05)$ (Figure 1B). Representative FACS data are shown in Figure 1C.

IQM decreased the expression of CCL17 and CCL22 in B16F10 melanoma. To investigate the immunomodulatory mechanisms responsible for the decrease of Tregs, we evaluated the immunomodulatory effects of topical IQM. We determined the mRNA expression of type 1 and $2 \mathrm{~T}$ helper cell (Th1/Th2)related chemokines and CCL2, which is a key chemokine for the induction of antitumor effects in B16F10 melanoma (3, 4, $7,10)$, in tumors from IQM-treated and untreated mice by using qRT-PCR. Compared to untreated mice, topical IQM significantly decreased the mRNA expression of Th2/Tregrelated chemokines (CCL17 and CCL22) in the tumor microenvironment (Figure 2A). The mRNA expression of Th1 chemokines (CXCL9, CXCL10 and CXCL11) or CCL2 was not significantly augmented (Figure $2 \mathrm{~A}$ ).

IQM decreased CCL22 production from CD11b ${ }^{+}$TAMs in B16F10 melanoma. To validate the immunomodulatory effects of topical IQM on cancer stroma cells, we examined the effects of IQM on chemokine production from CD11 ${ }^{+}$ TAMs, which are the main population of tumor-infiltrating lymphocytes (TILs) in B16F10 melanoma. CD11b ${ }^{+}$cells isolated from IQM-treated and untreated tumors were cultured for $48 \mathrm{~h}$ with LPS. The chemokines in the culture supernatant were then quantified by ELISA. Consistent with the IQM-induced decrease in CCL22 mRNA expression within the tumor, CCL22 production by these isolated $\mathrm{CD}_{11} \mathrm{~b}^{+}$cells was also significantly decreased by IQM treatment as compared to that of $\mathrm{CD}_{11 \mathrm{~b}^{+}}$cells from untreated tumors $(p<0.05)$ (Figure $2 \mathrm{~B})$. There was no significant difference in the production of CXCL10 from $\mathrm{CD}_{11} \mathrm{~b}^{+}$cells of IQM-treated and untreated tumors (Figure 2C). Unexpectedly, CCL17, CXCL9 and CXCL11 were not detected in the culture supernatants (data not shown).

IQM increased the expression of perforin and granzyme $B$ and $\mathrm{CDS}^{+} \mathrm{T}$ cell recruitment in B16F10 melanoma. To investigate the immunomodulatory mechanisms responsible for the antitumor immune response against B16F10 melanoma growth, we evaluated the immunomodulatory effects of topical IQM. We analyzed the mRNA expression of Th1/Th2-related cytokines (IFN- $\gamma, p 35$ and $I L-10)$ as well as cytotoxic molecules (perforin and granzyme B) in tumors from IQM-treated and untreated mice by using qRT-PCR. Compared to untreated mice, topical IQM significantly increased the mRNA expression of perforin and granzyme B in the tumor microenvironment $(p<0.05)$ (Figure 3A).

Next, we evaluated the immunomodulatory effects of topical IQM on the profiles of TILs in the tumors by flow cytometry. Whole tumor cells were analyzed $48 \mathrm{~h}$ after stimulation with or without topical IQM. As shown in Figure $3 \mathrm{~B}$, the main population of TILs was CD $11 \mathrm{~b}^{+}$TAMs. The percentage of $\mathrm{CD}^{+} \mathrm{T}$ cells within the $\mathrm{CD} 45^{+}$cell population was significantly increased by topical administration of IQM as compared to untreated mice $(p<0.05)$. In contrast, there was no significant difference in the number of $\mathrm{CD}^{+} \mathrm{T}$ cells 
A

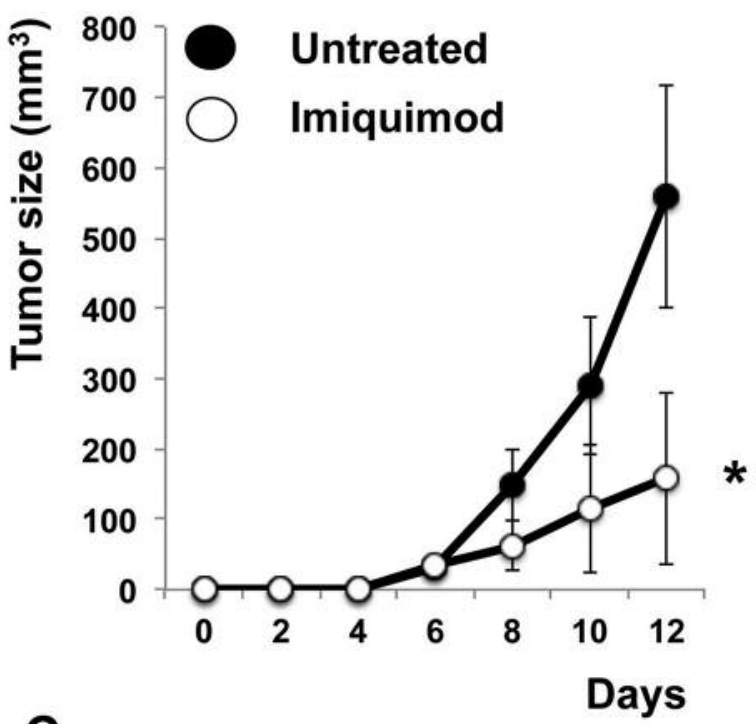

B

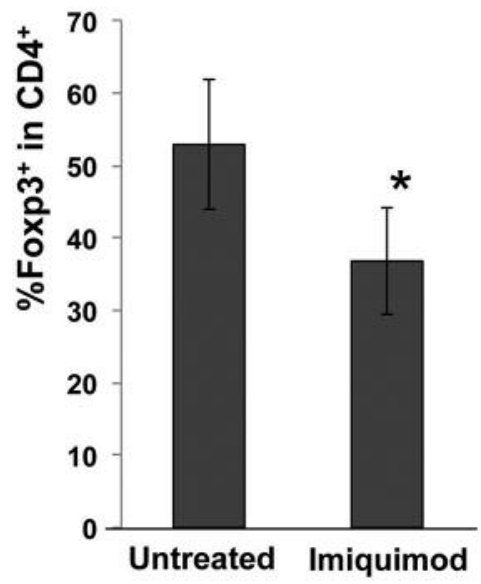

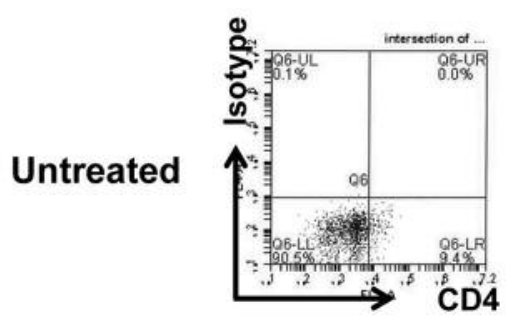
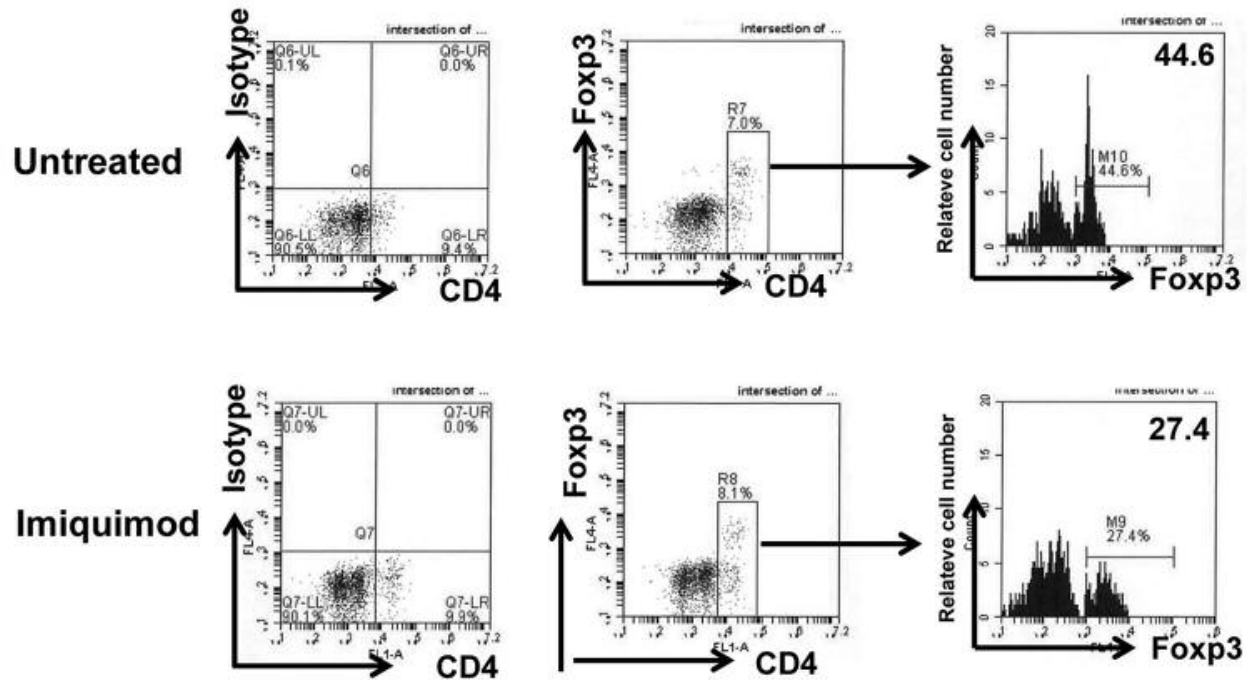

Figure 1. Therapeutic effect of topical IQM and its immunomodulatory effects in B16F10 melanoma. (A) B16F10 melanoma cells (100 $\mu$ l of $2 \times 10^{6}$ cells $/ \mathrm{ml}$ ) were subcutaneously injected into mice, and 5\% IQM cream was topically applied to the tumor mass on days 6, 8, 10 and 12. Untreated mice were used as a control. Tumor size was measured on the indicated days. The mean data from three independent experiments are shown ( $n=15$ for each treated group). (B) Flow cytometric analysis of Tregs in IQM-treated or untreated B16F10 melanomas. The intracellular expression of Foxp3 in $\mathrm{CD}^{+}$T cells in tumors was analyzed by FACS. Tumors measured approximately $7 \mathrm{~mm}$ in diameter. The mean percentage $( \pm S D)$ of Foxp $3^{+}$ cells among $\mathrm{CD}^{+} T$ cells obtained in six independent experiments is shown. (C) Representative data are shown.

or $\mathrm{CD} 11 \mathrm{~b}^{+}$cells within the $\mathrm{CD} 45^{+}$cell population in the presence or absence of IQM (Figure 3B). In addition, there was no significant difference in the percentage of PD-L1 ${ }^{+}$ cells within the $\mathrm{CD} 11 \mathrm{~b}^{+}$cell population in IQM-treated and untreated tumors (Figure 3C). There was no significant difference in the percentage of $\mathrm{CD} 69^{+}$cells and PD $-1^{+}$cells within the $\mathrm{CD}^{+}$and $\mathrm{CD} 8^{+} \mathrm{T}$ cell populations in IQMtreated and untreated tumors (Figure 3D).
Immunomodulatory effect of IQM on splenic $C D 11 b^{+}$cells in

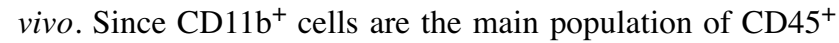
leukocytes in B16F10 melanoma, and since the number of

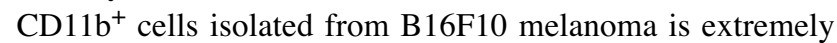
low, we next examined the immunomodulatory effects of topical IQM on splenic CD11 $\mathrm{b}^{+}$cells instead of TAMs. First, we evaluated the chemokine production of splenic CD11 $\mathrm{b}^{+}$ cells from tumor-bearing mice treated with or without IQM. 


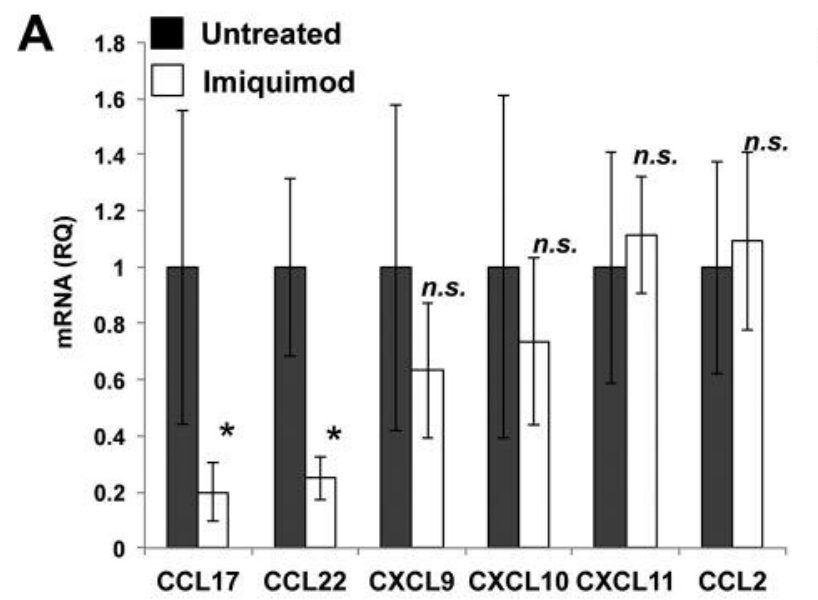

B
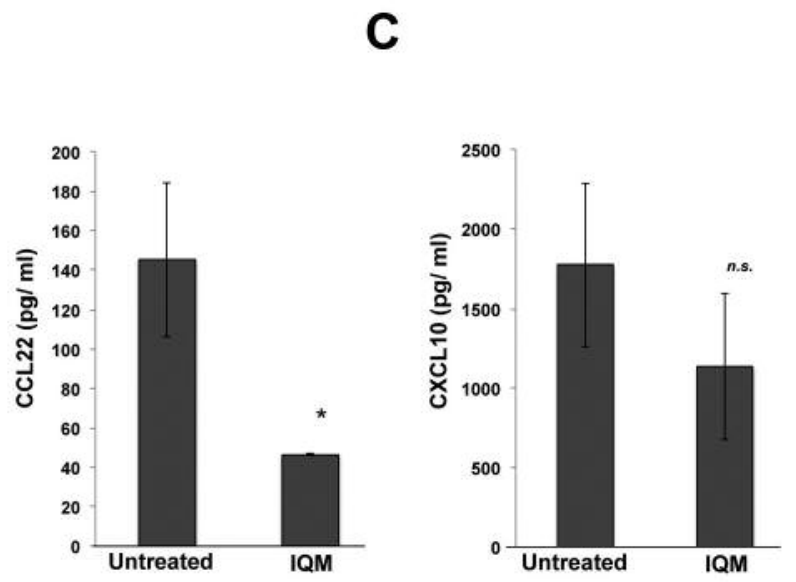

Figure 2. IQM modifies chemokine production from TAMs in B16F10 melanoma. (A) The mRNA expression levels of CCL17, CCL22, CXCL9, CXCL10, CXCL11 and CCL2 in B16F10 melanomas were analyzed by quantitative RT-PCR by the $\triangle \triangle C t$ method. One representative experiment of three is shown; error bars represent the SD within triplicates. (B) $C D 11 b^{+}$cells isolated from tumors of the indicated groups were stimulated with LPS $(0.5 \mu \mathrm{g} / \mathrm{ml})$, and chemokine production was measured by ELISA. One representative experiment of three is shown; error bars represent the $S D$ of triplicate experiments. ${ }^{*} p<0.05 ;$ n.s., not significant.

After isolation, these CD11b cells were cultured for $48 \mathrm{~h}$ with LPS, and then the chemokines in the culture supernatant were quantified by ELISA. In parallel with the IQM-induced decrease in CCL22 production from tumor-derived CD11b ${ }^{+}$ cells, CCL22 production from splenic CD11 $\mathrm{b}^{+}$cells was also significantly decreased by topical IQM as compared to that in cells from untreated tumors $(p<0.05)$ (Figure 4$)$. In addition, in vitro TLR7 agonist stimulation significantly decreased CCL22 production $(p<0.05)$, which suggests that IQM directly suppresses CCL22 production from splenic $\mathrm{CD}_{11 b^{+}}$cells. CXCL10 production was also decreased, although the decrease was limited (Figure 4).

IQM did not affect the suppressive function of splenic $\mathrm{CD}_{11 b^{+}}$cells in B16F10 melanoma-bearing hosts. To evaluate the suppressive function of splenic CD11 $\mathrm{b}^{+}$cells in

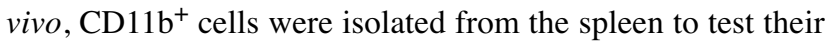
capacity to suppress $\mathrm{T}$ cell proliferation. Isolated $\mathrm{CD} 11 \mathrm{~b}^{+}$ cells were co-cultured with syngeneic $\mathrm{CD}^{+} \mathrm{T}$ cells and allogeneic BMDCs as stimulators. There was no significant difference in suppressive function of splenic CD11b ${ }^{+}$cells in each group (Figure 4C).

Splenic CD11 $b^{+}$cells modulated B16F10 melanoma growth in vivo. To determine if the topical IQM-induced decrease in CCL22 production from splenic CD11b ${ }^{+}$cells (Figure 4A) and suppression of Treg recruitment to the tumor (Figure 1B) influence tumor cell growth, we investigated the effects of splenic $\mathrm{CD} 11 \mathrm{~b}^{+}$cells on $\mathrm{B} 16 \mathrm{~F} 10$ melanoma growth in vivo. We isolated $\mathrm{CD}_{11} \mathrm{~b}^{+}$cells from the spleens of IQM-treated or untreated mice and implanted them together with $\mathrm{B} 16 \mathrm{~F} 10$ melanoma cells. B16F10 melanoma growth was accelerated by splenic $\mathrm{CD} 11 \mathrm{~b}^{+}$cells from untreated mice but was inhibited by

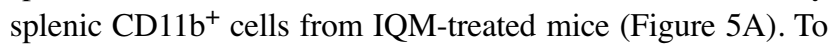
confirm the effects of splenic $\mathrm{CD}_{11 \mathrm{~b}^{+}}$cells on Treg recruitment, we evaluate the number of Tregs in the tumors by flow cytometry. The percentage of Foxp $3^{+}$Tregs within the $\mathrm{CD} 4^{+} \mathrm{T}$ cell population was significantly decreased by splenic $\mathrm{CD} 11 \mathrm{~b}^{+}$cells from IQM-treated mice $(p<0.05)$ (Figure 5B). Representative FACS data are shown in Figure 5C.

CCL22 Ab significantly suppressed splenic CD11 $b^{+}$cells and modulated B16F10 melanoma growth in vivo. To determine if the decrease in Tregs is due to decreased CCL22

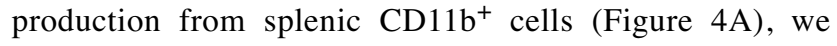
investigated the effects of anti-CCL22 Ab on B16F10 melanoma growth in the presence of splenic CD11 $\mathrm{b}^{+}$cells in vivo. We isolated splenic $\mathrm{CD}_{11 \mathrm{~b}^{+}}$cells from untreated mice and implanted them together with B16F10 melanoma. We then treated $\mathrm{B} 16 \mathrm{~F} 10$ melanomas $(5 \mathrm{~mm}$ in diameter) on the backs of mice by intratumoral injection of anti-CCL22 $\mathrm{Ab}$ (0.1 mg/mouse) or control IgG $(0.1 \mathrm{mg} /$ mouse $)$. B16F10 melanoma growth was inhibited by intratumoral injection of anti-CCL22 $\mathrm{Ab}$ (Figure 5D). To confirm the effects of intratumoral injection of anti-CCL22 $\mathrm{Ab}$ on Treg recruitment, we evaluated the number of Tregs in the tumors by flow cytometry. The percentage of Foxp $3^{+}$Tregs within the $\mathrm{CD} 4^{+} \mathrm{T}$ cell population was significantly decreased by intratumoral injection of anti-CCL22 Ab $(p<0.05)$ (Figure 5E). Representative FACS data are shown in Figure 5F. 
A

B
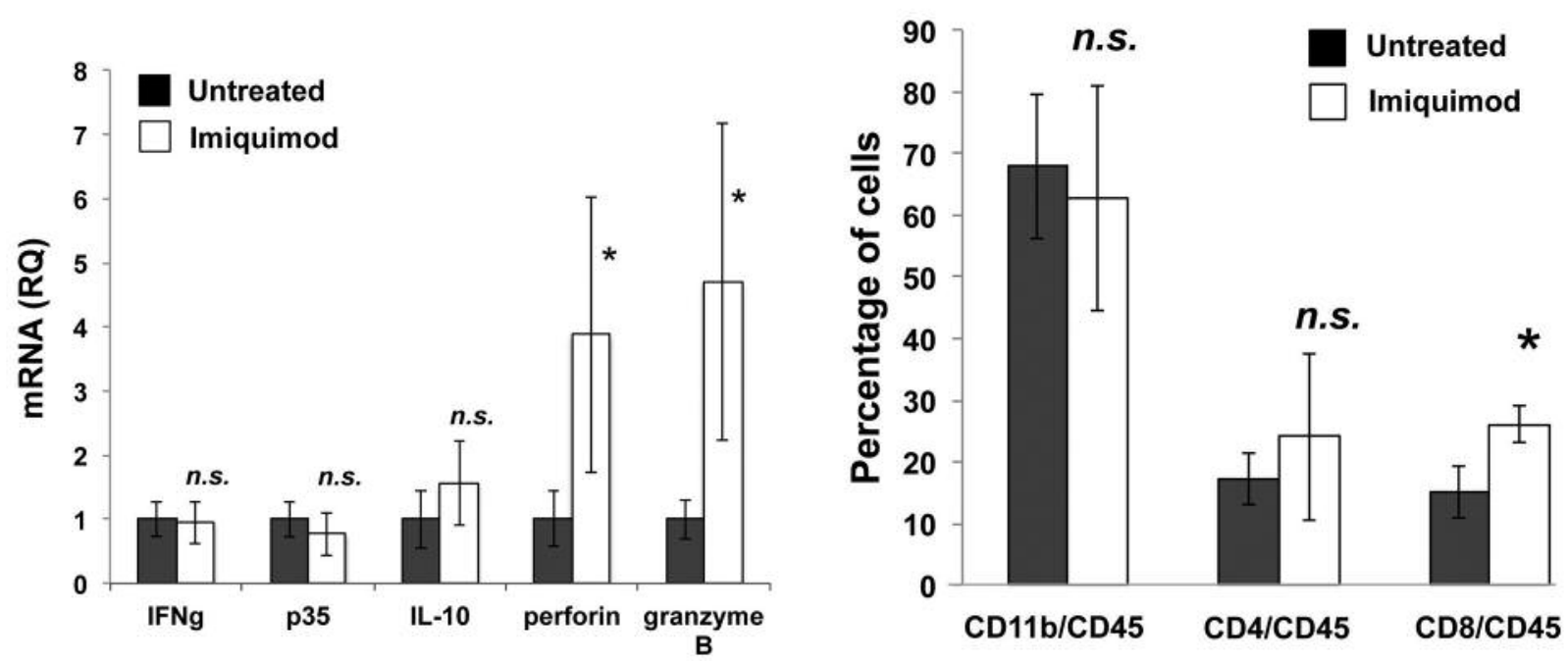

C

D
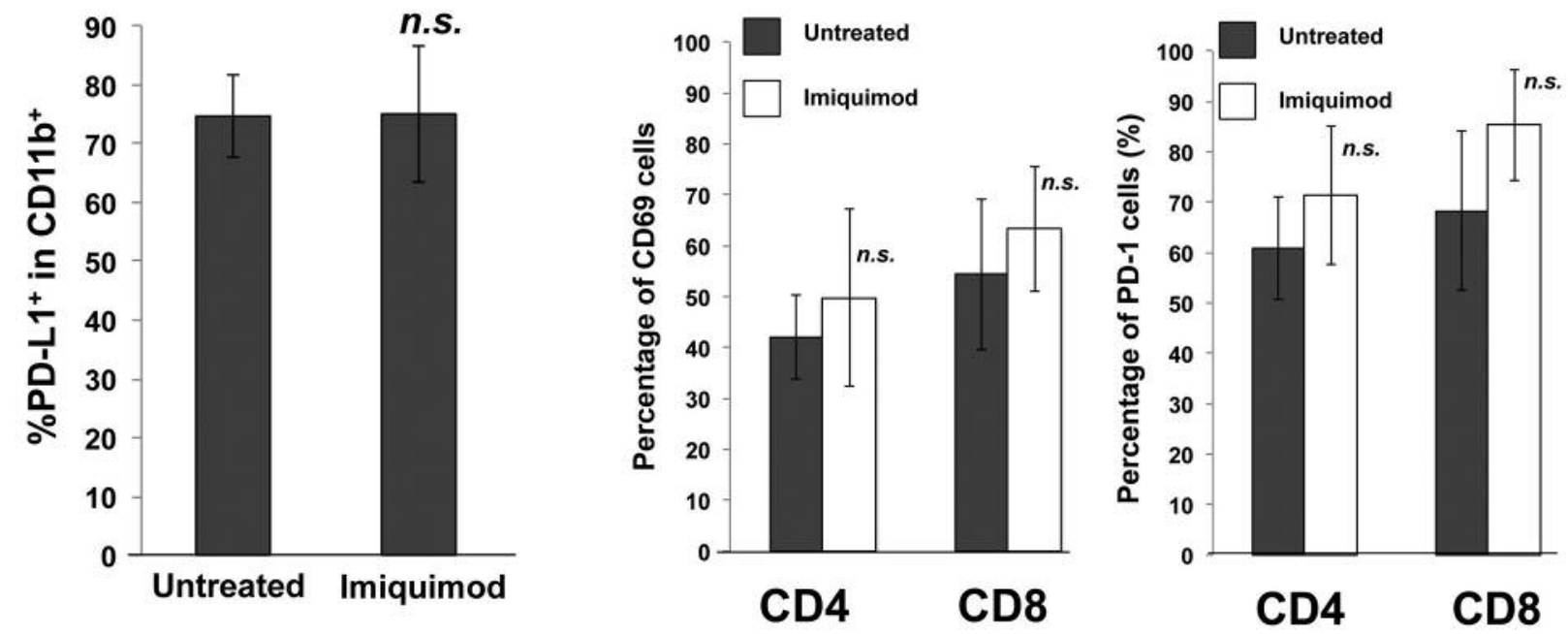

Figure 3. IQM modifies the profiles of TILs in B16F10 melanoma. (A) The mRNA expression of IFN- $\gamma, p 35$, IL-10, perforin and granzyme B in $B 16 F 10$ melanoma was analyzed by quantitative RT-PCR by using the $\triangle \Delta C t$ method. One representative experiment of three is shown; error bars represent the SD of triplicate experiments. (B-D) Flow cytometric analysis of TILs in IQM-treated or untreated B16 melanomas. The surface expression of $C D 45$ on $C D 11 b^{+}, C D 4^{+}$or $C D 8^{+}$cells $(B)$, the surface expression of $P D-L 1$ on $C D 11 b^{+}$cells $(C)$ and the surface expression of $C D 69$ and $P D-1$ on $\mathrm{CD}^{+}$or $C D 8^{+} T$ cells $(D)$ in tumors was analyzed by FACS. Tumors measured approximately $7 \mathrm{~mm}$ in diameter (B-D). The mean percentage $( \pm S D)$ of marker-positive cells among $C D 4^{+}$cells, $C D 8^{+}$cells, $C D 11 b^{+}$cells or $C D 45^{+}$cells obtained in six independent experiments is shown. ${ }^{*} p<0.05$; n.s., not significant.

\section{Discussion}

TAMs and circulating immature myeloid cells, so-called myeloid-derived suppressor cells (MDSCs), play crucial roles in maintaining the tumor microenvironment together with Tregs (16-20). In the differentiation cascade of myeloid cells in the tumor-bearing host (21), TAMs are derived from
Ly6C $\mathrm{C}^{+}$monocytes (monocytic MDSCs) in the blood (21). The majority of TAMs are alternatively activated M2 macrophages, which represent the main population of inflammatory cells in solid tumors (21-24). Since some populations of TAMs are derived from MDSCs, the mechanisms of suppressive activities of TAMs are similar to those of MDSCs $(16,17,21,22,25)$. For instance, the 
A
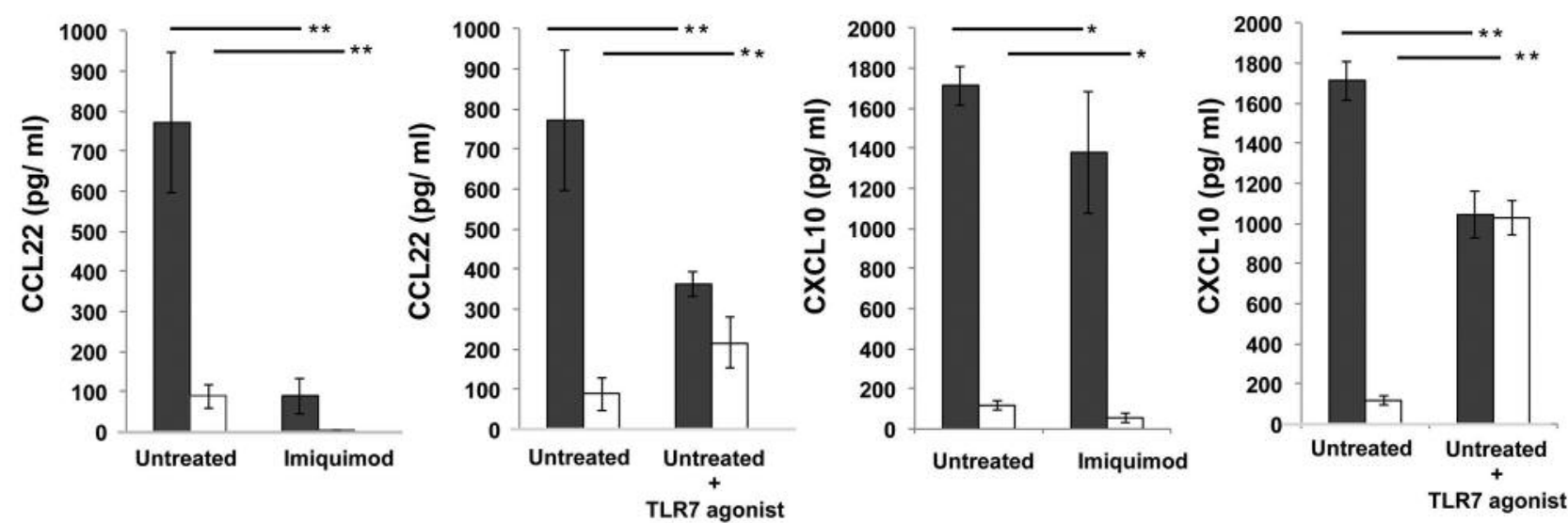

B
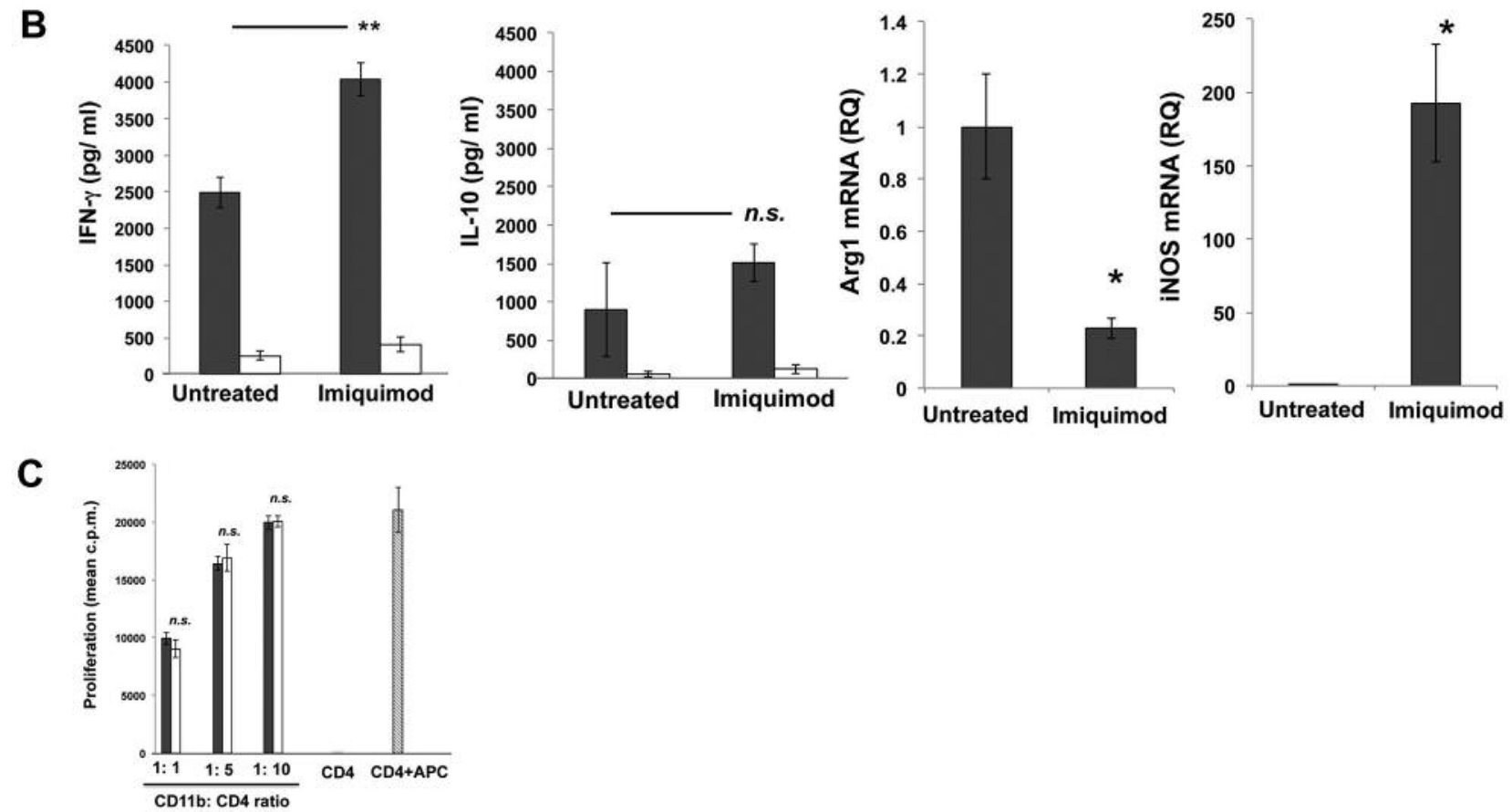

Figure 4. IQM and TLR7 agonist modifies chemokine production from CD11 ${ }^{+}$cells in B16F10 melanoma. (A) Splenic CD11b ${ }^{+}$cells were isolated from untreated or IQM-treated mice. The cells were stimulated with or without LPS and TLR7 agonist (0.5 $\mu$ g/ml), and cytokine production was measured by ELISAs. One representative experiment of three is shown; error bars represent the $S D$ of triplicate experiments. (B) Quantitative realtime PCR for validation of the DNA microarray results was conducted for each cytokine, arginase 1 (Arg1) and inducible nitric oxide synthase (iNOS) and normalization against GAPDH using the $\triangle \triangle C$ t method. Data from each donor were obtained in triplicate assays, and then the mean $\pm S D$ was calculated. Means of data from at least three independent experiments are shown. (C) Indicated numbers of splenic CD11 ${ }^{+}$cells from different mouse groups were co-cultured with syngeneic CD4+ T cells and allogeneic BMDCs for $72 \mathrm{~h} . \mathrm{T}$ cell proliferation was determined by $3 \mathrm{H}$-thymidine incorporation and expressed as counts per minute (cpm). One representative experiment of two is shown.

suppressive function of TAMs and MDSCs is partly mediated by the metabolism of $\mathrm{L}$-arginine, which is determined by the expression of high levels of arginase 1 and inducible nitric oxide synthase, both of which inhibit $\mathrm{T}$ cell proliferation $(16,17,21,22,25)$. In addition, both TAMs and
MDSCs produce Treg-related chemokines to recruit Tregs to the tumor microenvironment $(16,17,21,22,25-27)$. Moreover, TAMs and MDSCs express immune checkpoint molecules, such as PD-L1 and B7-1, to suppress the activities of effector memory $\mathrm{T}$ cells in the tumor 

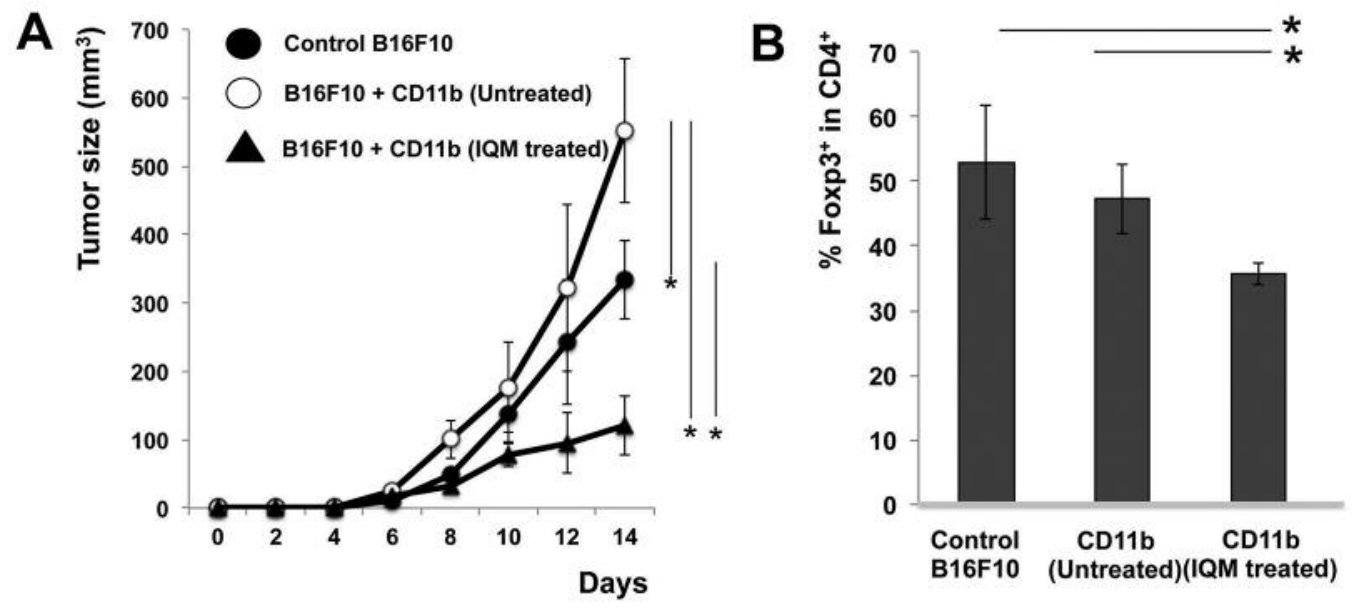

C
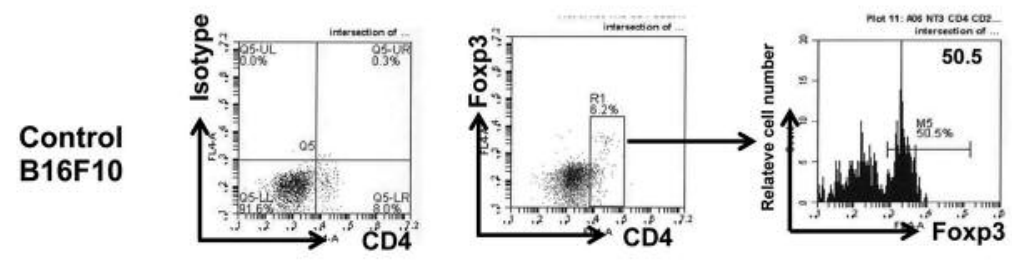

CD11b (Untreated)
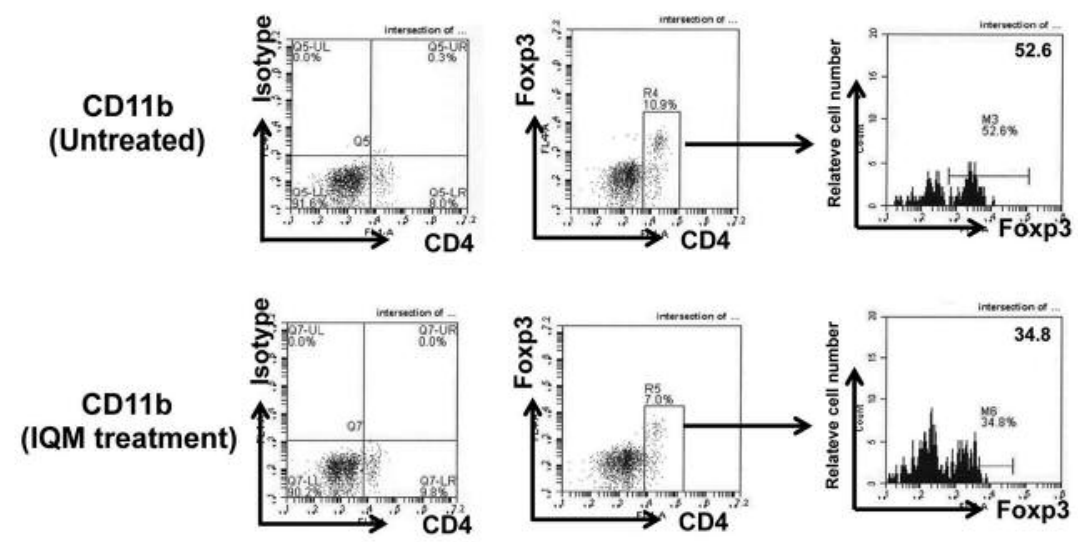

D
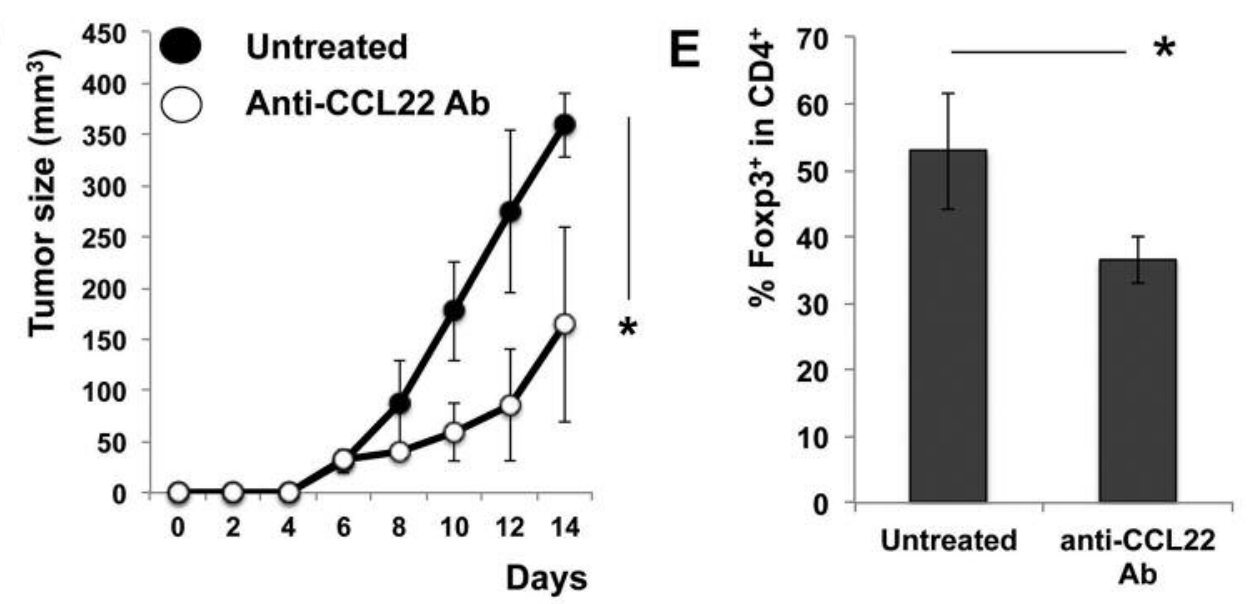

Figure 5. Continued 

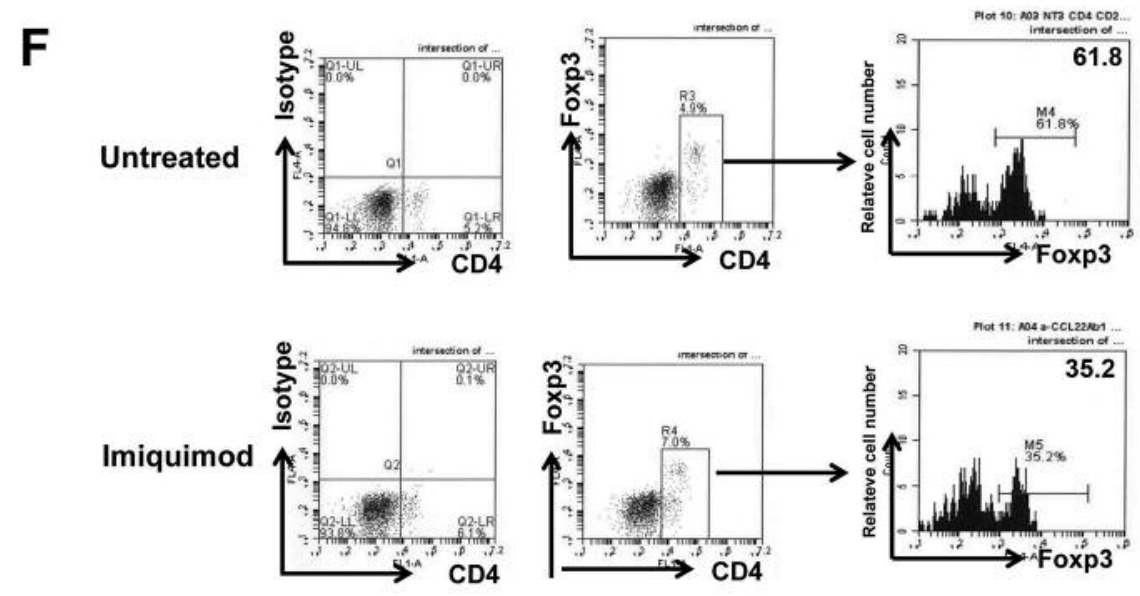

Figure 5. Topically-administered IQM modifies the profiles of splenic CD11b ${ }^{+}$myeloid cells in B16F10 melanoma. (A) B16F10 melanoma cells $\left(100 \mu \mathrm{l}, 2 \times 10^{6} \mathrm{cells} / \mathrm{ml}\right)$ were subcutaneously injected into mice with or without splenic CDI1 $b^{+}$cells isolated from untreated or IQM-treated mice ( $n=12$ for each treated group). (B) Flow cytometric analysis of Tregs in B16F10 melanomas injected with splenic CD11b ${ }^{+}$cells isolated from untreated or IQM-treated mice $(n=6)$. (C) Representative FACS data of Figure $5 B$ are shown. (D) B16F10 melanoma cells $\left(100 \mu l, 2 \times 10^{6} \mathrm{cells} / \mathrm{ml}\right)$ were subcutaneously injected into mice with splenic CD11b+ cells isolated from untreated mice, with or without anti-CCL22 Ab (n=6). (E) Flow cytometric analysis of Tregs in B16F10 melanomas injected with splenic CD11 ${ }^{+}$cells isolated from untreated mice with or without anti-CCL22 $A b(n=6) .(F)$ Representative FACS illustration of Figure $5 E$ is shown. ${ }^{*} p<0.05$; n.s., not significant.

microenvironment $(15,25)$. Notably, TAMs are a heterogeneous population of cells that could be polarized to M1-like macrophages in the tumor microenvironment (21). Therefore, TAMs could be a promising immunotherapy target in the treatment of melanoma $(13,28)$.

In the present study, we show the immunomodulatory effects of IQM on TAMs and splenic CD11 ${ }^{+}$cells, leading to the inhibition of $\mathrm{B} 16 \mathrm{~F} 10$ melanoma growth in vivo. As Lawrence et al. have reviewed (21), we demonstrated that the production of several chemokines (CCL17, CCL22, CXCL9, CXCL10 and CXCL11) of splenic CD11b ${ }^{+}$cells resembled those of TAMs. Subsequently, we investigated the

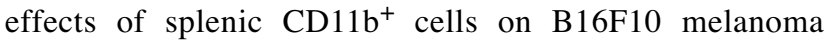
growth in vivo. B16F10 melanoma growth was accelerated by splenic $\mathrm{CD} 11 \mathrm{~b}^{+}$cells from untreated mice but inhibited by splenic $\mathrm{CD} 11 \mathrm{~b}^{+}$cells from IQM-treated mice. Moreover, Foxp $^{+}$Treg recruitment to the tumor was significantly decreased when splenic $\mathrm{CD}_{11} \mathrm{~b}^{+}$cells from IQM-treated mice were used. Notably, CCL22 production by both tumorderived and splenic $\mathrm{CD}_{11 \mathrm{~b}^{+}}$cells was significantly decreased by IQM treatment compared to that of CD11b cells from untreated hosts. As previous reports suggested, CCL22 diverts Tregs and controls B16F10 melanoma growth (13), suggesting the reduction of tumor-derived CCL22 could suppress melanoma growth. Indeed, the blockade of CCL22 by intratumoral injection of anti-CCL22 Ab significantly inhibited Treg recruitment, leading to inhibition of B16F10 melanoma growth in vivo. We also evaluated the
mRNA expression of CCL2, which is a known ligand of CCR4 and reported to increase in advanced melanoma in an experimental murine model (14). Unlike CCL22 mRNA expression, IQM treatment did not affect the expression of CCL2 mRNA on B16F10 melanoma. These reports suggested that inhibition of tumor-derived chemokines could be a therapeutic target for melanoma by the reduction of Treg recruitment at the tumor site.

Tregs play a crucial role in maintaining peripheral tolerance that actively suppresses effector T cells (24). Tregs maintain the immunosuppressive microenvironment and promote tumor growth together with TAMs at the tumor site (10, 24, 29). Indeed, both MDSCs, which are known as immunosuppressive immature macrophages, and Tregs determine the prognosis of advanced melanoma patients (30). In addition, Mahnke et al. reported that Treg depletion by denileukin diftitox $\left(\right.$ Ontak $^{\circledR}$, Eisai Medical Research, Tokyo, Japan) in melanoma patients resulted in the substantial development of antigen-specific $\mathrm{CD}^{+} \mathrm{T}$ cells in vaccinated individuals (31). Another report also suggested that Treg depletion had a significant clinical effect in unresectable stage IV melanoma patients (32). These reports suggested that Treg depletion could be a supportive therapy for human melanoma.

The therapeutic effect of a TLR7 agonist (including IQM) or TLR7/8 dual agonist on melanoma growth in vivo has been previously reported $(2,4,7,33)$. These reports revealed the immunostimulatory effect of these agonists through 
effector cells, such as $\mathrm{CD}^{+} \mathrm{T}$ cells, plasmacytoid DCs or M1 macrophages, and decrease the Tregs and MDSC in the tumor lesion, leading to the inhibition of B 16F10 melanoma growth in vivo. In addition, more recently, several crinical trial has proved the antitumor effects of IQM against melanoma in situ $(34,35)$. In our present study, we further elucidated the immunomodulatory effect of IQM on TAMs and splenic $\mathrm{CD}_{11} \mathrm{~b}^{+}$cells during melanoma growth in vivo, especially focusing on the production of Treg-related chemokines and effector T cells-related chemokines. Indeed, we showed that topical IQM reduced the immunosuppressive function of TAMs in B16F10 melanomas and even promoted the subpopulation of splenic $\mathrm{CD} 11 \mathrm{~b}^{+}$cells that inhibited B16F10 melanoma growth. Our present study revealed another possible mechanism of the antitumor effect of IQM.

\section{Acknowledgements}

This study was supported in part by grants-in-aid for scientific research from the Japan Society for the Promotion of Science (Nos. 23791249 and 25461682). The 5\% imiquimod cream was a kind gift from Mochida Pharmaceutical Co., Ltd (Tokyo, Japan).

\section{Conflicts of Interest}

The Authors declare no conflicts of interest.

\section{Funding}

This study was supported, in part, by grants-in-aid for scientific research from the Japan Society for the Promotion of Science (Nos. 23791249, 25461682 and 16K10143).

\section{References}

1 Adlard AL, Dovedi SJ, Telfer BA, Koga-Yamakawa E, Pollard C, Honeychurch J, Illidge TM, Murata M, Robinson DT, Jewsbury PJ, Wilkinson RW and Stratford IJ: A novel systemically administered Toll-like receptor 7 agonist potentiates the effect of ionizing radiation in murine solid tumor models. Int J Cancer 135: 820-829, 2014.

2 Drobits B, Holcmann M, Amberg N, Swiecki M, Grundtner R, Hammer M, Colonna M and Sibilia M: Imiquimod clears tumors in mice independent of adaptive immunity by converting pDCs into tumor-killing effector cells. J Clin Invest 122: 575-585, 2012 .

3 Ma F, Zhang J, Zhang J and Zhang C: The TLR7 agonists imiquimod and gardiquimod improve DC-based immunotherapy for melanoma in mice. Cell Mol Immunol 7: 381-388, 2010.

4 Redondo P, del Olmo J, Lopez-Diaz de Cerio A, noges S, Marquina M, Melero I and Bendandi M: Imiquimod enhances the systemic immunity attained by local cryosurgery destruction of melanoma lesions. J Invest Dermatol 127: 1673-1680, 2007.

5 Fujita M, Kohanbash G, Fellows-Mayle W, Hamilton RL, Komohara Y, Decker SA, Ohlfest JR and Okada H: COX-2 blockade suppresses gliomagenesis by inhibiting myeloidderived suppressor cells. Cancer Res 71: 2664-2674, 2011.
6 Zhou W, Ke SQ, Huang Z, Flavahan W, Fang X, Paul J, Wu L, Sloan AE, McLendon RE, Li X, Rich JN and Bao S: Periostin secreted by glioblastoma stem cells recruits M2 tumourassociated macrophages and promotes malignant growth. Nat Cell Biol 17: 170-182, 2015.

7 Singh M, Khong H, Dai Z, Huang XF, Wargo JA, Cooper ZA, Vasilakos JP, Hwu P and Overwijk WW: Effective innate and adaptive antimelanoma immunity through localized TLR7/8 activation. J Immunol 193: 4722-4731, 2014.

8 Adams S, O'Neill DW, Nonaka D, Hardin E, Chiriboga L, Siu K, Cruz CM, Angiulli A, Angiulli F, Ritter E, Holman RM, Shapiro RL, Berman RS, Berner N, Shao Y, Manches O, Pan L, Venhaus RR, Hoffman EW, Jungbluth A, Gnjatic S, Old L, Pavlick AC and Bhardwaj N: Immunization of malignant melanoma patients with full-length NY-ESO-1 protein using TLR7 agonist imiquimod as vaccine adjuvant. J Immunol 181: 776-784, 2008.

9 Turza K, Dengel LT, Harris RC, Patterson JW, White K, Grosh WW and Slingluff CL Jr.: Effectiveness of imiquimod limited to dermal melanoma metastases, with simultaneous resistance of subcutaneous metastasis. J Cutan Pathol 37: 9498, 2010.

10 van den Boorn JG, Konijnenberg D, Tjin EP, Picavet DI, Meeuwenoord NJ, Filippov DV, van der Veen JP, Bos JD, Melief $\mathrm{CJ}$ and Luiten RM: Effective melanoma immunotherapy in mice by the skin-depigmenting agent monobenzone and the adjuvants imiquimod and CpG. PloS One 5: e10626, 2010.

11 Stone GW, Barzee S, Snarsky V, Santucci C, Tran B, Langer R, Zugates GT, Anderson DG and Kornbluth RS: Nanoparticledelivered multimeric soluble CD40L DNA combined with TollLike Receptor agonists as a treatment for melanoma. PloS One 4: e7334, 2009.

12 Klarquist J, Tobin K, Farhangi Oskuei P, Henning SW, Fernandez MF, Dellacecca ER, Navarro FC, Eby JM, Chatterjee S, Mehrotra S, Clark JI and Le Poole IC: Ccl22 Diverts T Regulatory Cells and Controls the Growth of Melanoma. Cancer Res 76: 6230-6240, 2016.

13 Kakizaki A, Fujimura T, Furudate S, Kambayashi Y, Yamauchi $\mathrm{T}$, Yagita $\mathrm{H}$ and Aiba $\mathrm{S}$ : Immunomodulatory effect of peritumorally administered interferon-beta on melanoma through tumor-associated macrophages. Oncoimmunology 4 : e1047584, 2015.

14 Kimpfler S, Sevko A, Ring S, Falk C, Osen W, Frank K, Kato M, Mahnke K, Schadendorf D and Umansky V: Skin melanoma development in ret transgenic mice despite the depletion of $\mathrm{CD} 25^{+}{ }^{+}$oxp $3+$ regulatory $\mathrm{T}$ cells in lymphoid organs. J Immunol 183: 6330-6337, 2009.

15 Fujimura T, Ring S, Umansky V, Mahnke K and Enk AH: Regulatory $\mathrm{T}$ cells stimulate B7-H1 expression in myeloidderived suppressor cells in ret melanomas. J Invest Dermatol 132: 1239-1246, 2012.

16 Gabrilovich DI and Nagaraj S: Myeloid-derived suppressor cells as regulators of the immune system. Nat Rev Immunol 9: 162174, 2009.

17 Fujimura T, Mahnke K and Enk AH: Myeloid derived suppressor cells and their role in tolerance induction in cancer. J Dermatol Sci 59: 1-6, 2010.

18 Italiani $\mathrm{P}$ and Boraschi D: From Monocytes to M1/M2 Macrophages: Phenotypical vs. Functional Differentiation. Front Immunol 5: 514, 2014. 
19 Romano E, Kusio-Kobialka M, Foukas PG, Baumgaertner P, Meyer C, Ballabeni P, Michielin O, Weide B, Romero P and Speiser DE: Ipilimumab-dependent cell-mediated cytotoxicity of regulatory $\mathrm{T}$ cells ex vivo by nonclassical monocytes in melanoma patients. Proc Natl Acad Sci USA 112: 6140-6145, 2015.

20 Tiemessen MM, Jagger AL, Evans HG, van Herwijnen MJ, John $\mathrm{S}$ and Taams LS: $\mathrm{CD} 4{ }^{+} \mathrm{CD} 25^{+}{ }^{+}$Foxp $3{ }^{+}$regulatory $\mathrm{T}$ cells induce alternative activation of human monocytes/macrophages. Proc Natl Acad Sci USA 104: 19446-19451, 2007.

21 Lawrence $\mathrm{T}$ and Natoli G: Transcriptional regulation of macrophage polarization: enabling diversity with identity. Nat Rev Immunol 11: 750-761, 2011.

22 Fujimura T, Kakizaki A, Furudate S, Kambayashi Y and Aiba S: Tumor-associated macrophages in skin: how to treat their heterogeneity and plasticity. J Dermatol Sci 83: 167-173, 2016.

23 Lewis CE and Pollard JW: Distinct role of macrophages in different tumor microenvironments. Cancer Res 66: 605-612, 2006.

24 Tang X, Mo C, Wang Y, Wei D and Xiao H: Anti-tumour strategies aiming to target tumour-associated macrophages. Immunology 138: 93-104, 2013.

25 Noy R and Pollard JW: Tumor-associated macrophages: from mechanisms to therapy. Immunity 41: 49-61, 2014.

26 Fujimura T, Kambayashi Y, Furudate S, Asano M, Kakizaki A and Aiba S: Receptor Activator of NF-kappaB Ligand Promotes the Production of CCL17 from RANK ${ }^{+}$M2 Macrophages. J Invest Dermatol 135: 2884-2887, 2015.

27 Muthuswamy R, Wang L, Pitteroff J, Gingrich JR and Kalinski P: Combination of IFNalpha and poly-I:C reprograms bladder cancer microenvironment for enhanced CTL attraction. J Immunother Cancer 3: 6, 2015.

28 Fujimura T, Hidaka T, Kambayashi Y, Furudate S, Kakizaki A, Tono H, Tsukada A, Haga T, Hashimoto A, Morimoto R, Yamaguchi T, Takano T and Aiba S: Phase I study of nivolumab combined with IFN- $\beta$ for patients with advanced melanoma. Oncotarget 2017, in press. doi: 10.18632/oncotarget.17090. [Epub ahead of print]

29 Baksh K and Weber J: Immune checkpoint protein inhibition for cancer: preclinical justification for CTLA-4 and PD-1 blockade and new combinations. Semin Oncol 42: 363-377, 2015.
30 Allavena P, Sica A, Solinas G, Porta C and Mantovani A: The inflammatory micro-environment in tumor progression: the role of tumor-associated macrophages. Crit Rev Oncol Hematol 66: 1-9, 2008.

31 Mahnke K, Schonfeld K, Fondel S, Ring S, Karakhanova S, Wiedemeyer K, Bedke T, Johnson TS, Storn V, Schallenberg S and Enk AH: Depletion of $\mathrm{CD} 4{ }^{+} \mathrm{CD} 25^{+}$human regulatory $\mathrm{T}$ cells in vivo: kinetics of Treg depletion and alterations in immune functions in vivo and in vitro. Int J Cancer 120: 27232733, 2007

32 Telang S, Rasku MA, Clem AL, Carter K, Klarer AC, Badger WR, Milam RA, Rai SN, Pan J, Gragg H, Clem BF, McMasters KM, Miller DM and Chesney J: Phase II trial of the regulatory $\mathrm{T}$ cell-depleting agent, denileukin diftitox, in patients with unresectable stage IV melanoma. BMC Cancer 11: 515, 2011.

33 Cho JH, Lee HJ, Ko HJ, Yoon BI, Choe J, Kim KC, Hahn TW, Han JA, Choi SS, Jung YM, Lee KH, Lee YS and Jung YJ: The TLR7 agonist imiquimod induces anti-cancer effects via autophagic cell death and enhances anti-tumoral and systemic immunity during radiotherapy for melanoma. Oncotarget 8 : 24932-24948, 2017.

34 Marsden JR, Fox R, Boota NM, Cook M, Wheatley K, Billingham LJ and Steven NM; NCRI Skin Cancer Clinical Studies Group, the U.K. Dermatology Clinical Trials Network and the LIMIT-1 Collaborative Group: Effect of topical imiquimod as primary treatment for lentigo maligna: the LIMIT1 study. Br J Dermatol, in press 2016. doi: 10.1111/bjd.15112. [Epub ahead of print]

35 Mora AN, Karia PS and Nguyen BM: A quantitative systematic review of the efficacy of imiquimod monotherapy for lentigo maligna and an analysis of factors that affect tumor clearance. $J$ Am Acad Dermatol 73: 205-212, 2015.

Received April 26, 2017

Revised May 13, 2017

Accepted May 16, 2017 